\title{
Jackiw-Teitelboim supergravity, minimal strings, and matrix models
}

\author{
Clifford V. Johnson* \\ Department of Physics and Astronomy, University of Southern California, \\ Los Angeles, California 90089-0484, USA
}

(Received 23 December 2020; accepted 4 January 2021; published 19 February 2021)

\begin{abstract}
It is proposed that a family of Jackiw-Teitelboim supergravites, recently discussed in connection with matrix models by Stanford and Witten, can be given a complete definition, to all orders in the topological expansion and beyond, in terms of a specific combination of minimal string theories. This construction defines nonperturbative physics for the supergravity that is well defined and stable. The minimal models come from double-scaled complex matrix models and correspond to the cases $(2 \Gamma+1,2)$ in the AltlandZirnbauer $(\boldsymbol{\alpha}, \boldsymbol{\beta})$ classification of random matrix ensembles, where $\Gamma$ is a parameter. A central role is played by a nonlinear "string equation" that naturally incorporates $\Gamma$, usually taken to be an integer, counting, e.g., D-branes in the minimal models. Here, half-integer $\Gamma$ also has an interpretation. In fact, $\Gamma= \pm \frac{1}{2}$ yields the cases $(0,2)$ and $(2,2)$ that were shown by Stanford and Witten to have very special properties. These features are manifest in this definition because the relevant solutions of the string equation have special properties for $\Gamma= \pm \frac{1}{2}$. Additional special features for other half-integer $\Gamma$ 's suggest new surprises in the supergravity models.
\end{abstract}

DOI: 10.1103/PhysRevD.103.046012

\section{INTRODUCTION}

Recently, there has been renewed interest in JackiwTeitelboim (JT) gravity [1,2], a two-dimensional theory of gravity that emerges in various physical contexts, such as the study of the near-horizon dynamics of nearly extreme black holes [3,4], or as a partial gravitational dual of certain one-dimensional quantum mechanical systems pertinent to studies of condensed matter and quantum chaos. (For reviews, see Refs. [5,6].) In its own right, it is an arena for further developing understanding of the interplay between quantum mechanics, geometry, and topology.

The JT gravity partition function $Z(\beta)$ can be written (in a Euclidean presentation, where $\beta$ is the period of compact time) as a topological expansion summing contributions from constant negative curvature surfaces of genus $g$ (the number of handles) with a boundary of fixed length $\beta$.

The dynamics of the theory lives on the boundary, and it has a Schwarzian action [7-9]. The leading contribution, which comes from the $g=0$ (disc) topology, gives a result $Z_{0}(\beta)$, which can be written [10]

*johnson1@usc.edu

Published by the American Physical Society under the terms of the Creative Commons Attribution 4.0 International license. Further distribution of this work must maintain attribution to the author(s) and the published article's title, journal citation, and DOI. Funded by SCOAP.

$$
Z_{0}(\beta)=e^{S_{0}} \int d E \rho_{0}(E) e^{-\beta E}
$$

where $\rho_{0}(E)$ is a spectral density function. Here, $S_{0}$ is a constant proportional to $1 / G$, where $G$ is the Newton constant of the 2D gravity. (In fact, $S_{0}$ is the leading black hole entropy, if approaching this model from a nearhorizon dynamics perspective.) Here, it will be useful to define a parameter $\hbar=e^{-S_{0}}$, since it will naturally appear as a Planck constant in an important associated problem to be described shortly. The result for the disc spectral density is [11]

$$
\rho_{0}(E)=\frac{\gamma \sinh (2 \pi \sqrt{2 \gamma E})}{2 \pi^{2} \hbar}
$$

where $\hbar^{-1}$ will be absorbed into its definition henceforth. The value of $\gamma$ determines the units when relating a coupling in the Schwarzian to $\beta$. Here, $\gamma=\frac{1}{2}$ will be chosen.

JT gravity emerges [7-9,12] (at low energy) as a gravitational dual of certain 1D quantum systems that exhibit chaos, such as the Sachdev-Ye-Kitaev (SYK) model $[11,13,14]$, and various features of the chaotic dynamics suggest $[10,15,16]$ a relation to models of large- $N$ random matrix models. Then Saad, Shenker, and Stanford showed [17] that the entire topological expansion for JT gravity can be captured by a Hermitian matrix model in a certain "double-scaling" limit [18-21]. The double-scaled $1 / N$ expansion of the model gives a genus expansion in $2 \mathrm{D}$ surfaces $[22,23]$, and has its contributions at higher genera 
fully determined by a family of recursion relations [24-27] seeded by the disc spectral density $\rho_{0}(E)$, which was shown [17] to precisely match analogous features of JT gravity.

The original double-scaled matrix models [18-21] were used to define the sum over random surfaces that were the string world sheets in what have now come to be called "minimal string theories" [28]. While not itself a minimal string theory, JT gravity shares a number of features with them, and Ref. [17] suggests that JT gravity can be thought of as an infinite-order limit of minimal string theories. An (apparently) alternative picture, suggested in Ref. [29] and expanded upon in Ref. [30] (see also the recent Refs. [31,32]), is that JT gravity can be defined as a special interpolating flow among an infinite set of minimal string models. In fact, it will be proposed in Sec. II that the two suggestions are complementary, the latter being a refinement of the former.

The approach of constructing JT gravity out of minimal string models has certain advantages, as will be further demonstrated in this paper. The minimal models (arising from one-cut double-scaled matrix models) have a great deal of their physics readily accessible through an associated 1D problem, with the Hamiltonian

$$
\mathcal{H}=-\hbar^{2} \frac{\partial^{2}}{\partial x^{2}}+u(x)
$$

where the potential $u(x)$ satisfies a nonlinear ordinary differential equation (ODE) called a "string equation." This ODE supplies both perturbative and nonperturbative information for $u(x)$, and the spectral density of this system coincides with the spectral density of the doublescaled matrix model. Knowledge of the properties of the underlying string equation, especially nonperturbative features, can be used to infer properties of the JT model that are not apparent (or simply inaccessible) in the perturbative/recursive approach. For example, Ref. [30] used this insight to formulate a nonperturbative lowenergy completion of the JT matrix model of Ref. [17] that is free of the instabilities of the definition based on Hermitian matrix models.

In fact, the double-scaled matrix model description extends to other kinds of JT gravity. Various JT gravity and JT supergravity models were classified by Stanford and Witten [33] in terms of the underlying distinct random matrix ensembles available. They are either from the three $\beta$ ensembles à la Dyson-Wigner, or the seven $(\boldsymbol{\alpha}, \boldsymbol{\beta})$ ensembles in the Altland-Zirnbauer taxonomy [34]. In a series of nontrivial computations, the properties of the various matrix model recursion relations were checked against the corresponding JT gravity-type computations, and found to support the correspondence. Various other (mostly perturbative) features were uncovered in that work as well.

It is therefore natural to wonder if this wider class of JT gravities and their matrix models can be constructed out of

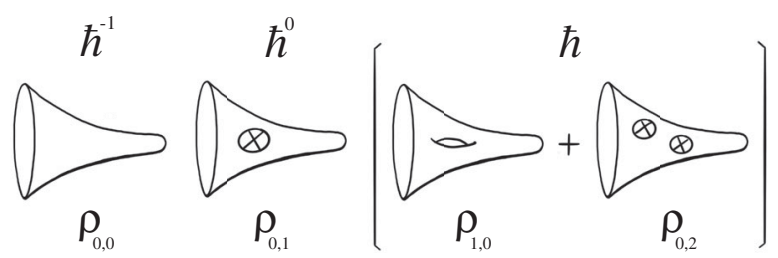

FIG. 1. Examples of perturbative contributions. A cross represents a crosscap insertion making a nonorientable surface.

appropriate kinds of minimal string models. Perhaps this could help to clarify their properties, or supply information about the nonperturbative sector, since the recursive methods of Refs. [17,33] are perturbative.

This paper will show that such constructions are possible, at least for some of the models. The focus will be directly on a set of JT supergravity theories (with and without time-reversal symmetry) that were discussed in Ref. [33], which is of relevance to $\mathcal{N}=1$ supersymmetric generalizations of the Schwarzian dynamics that arose in ordinary JT gravity, or the SYK model [35-40]. The disc spectral density of these models is $[33,41]$

$$
\rho_{0}(E)=\frac{\cosh (2 \pi \sqrt{E})}{\pi \hbar \sqrt{E}}=\frac{\rho_{0} \mathrm{SJT}}{\sqrt{2}}, \quad \text { SJT }
$$

where the factor of $\sqrt{2}$ gives the appropriate normalization for comparing the pertinent matrix ensembles to the supergravity path integral.

Before proceeding, it is prudent to enlarge the notation slightly. So far, the "0" subscript on the partition function and the corresponding density means that the result is at disc order in the genus expansion. In this broader class of theories, because of the possible inclusion of time-reversal symmetry, spacetime can be nonorientable, and so crosscaps should be included in the topological sum. Quantities such as the spectral density function will therefore be written perturbatively as

$$
\rho(E)=\sum_{g, b, c} \hbar^{2 g+c-1} \rho_{g, c}(E),
$$

where $g$ is the number of handles of the surface, and $c$ the number of crosscaps. So in this notation, $\rho_{0}(E) \equiv \rho_{0,0}(E)$. Henceforth, the powers of $\hbar$ will be explicitly included in the density, as was already done in, e.g., Eqs. (2) and (4). See Fig. 1 for the first three orders.

The core specific JT supergravity models in question are the $(\boldsymbol{\alpha}, \boldsymbol{\beta})=(\{0,1,2\}, 2)$ in the Altland-Zirnbauer classification. Many perturbative aspects of these models were constructed and exhibited in Ref. [33], including several important peculiarities, such as the vanishing of perturbative contributions to $\rho_{0}$ beyond the disc for the cases $\alpha \in\{0,2\}$. [As will become clear later, however, a broader 
class of models, $(2 \Gamma+1,2)$, will also be accessible via the methods of this paper too, and results for those will also be presented.]

The present work will supply a description of these supergravities in terms of an infinite interpolating set of minimal string models (of type 0A [42-47]), i.e., using the same ingredients presented in Ref. [30], but combining them differently (see Secs. II and IV). This will yield the spectral density [Eq. (4)] at disc level, in a way that generalizes the case for ordinary JT in an interesting (and suggestive) manner.

The approach used [the aforementioned string equations, and associated Hamiltonian problem (3) - see Sec. III for an introduction] will be quite complementary to the approaches of Refs. [17,33], and will have the advantage of making more manifest certain perturbative features of the models. For example, the type-0A minimal strings (via their defining string equation) have just the right properties (see Sec. III D) needed to yield the key leading (universal) $1 / \sqrt{E}$ dependence at low energy. Their (fractional) power-law rise with $E$ for higher energies collectively contributes to the overall exponential rise of the full model. As a further example, the vanishing (observed in Ref. [33]) of all perturbative contributions (beyond the leading terms) for $\alpha \in\{0,2\}$ will follow straightforwardly in this formalism, due to certain special properties of the string equations. (This is discussed in Sec. IVA.)

Moreover, the present approach allows a clear formulation of the full nonperturbative physics of the models that reduces to the disc result [Eq. (4)] for all $E$. This supplements and extends to all energies the nonperturbative aspects that were touched upon in Ref. [33], which applied mostly to the low-energy regime. Model behaviors will be displayed in Sec. IV C.

Another appealing feature of this paper's approach is that the formulation allows for a larger family of models$(2 \Gamma+1,2)$ in the classification scheme-to be cast into a single framework. The parameter $\Gamma$ is likely to be identified with the Stanford-Witten parameter $\nu$, counting "Ramond punctures" [33]. (See more discussion of this in Secs. V and IV B.) Again, certain perturbative features become manifest here. For example, the observation of Ref. [33] that only even numbers of punctures are allowed becomes a straightforward manifest feature of the formulation. The special cases $\alpha \in\{0,2\}$ correspond to $\Gamma= \pm \frac{1}{2}$, which is a special point of all type-0A solutions of the string equation. However, the string equation shows that there are other special features for more general half-integer $\Gamma$, suggesting that there are new aspects of these JT supergravities to be discovered in these cases. The present formulation supplies full nonperturbative physics here too. See Secs. IVA and IV C.

Section V presents some closing remarks and ideas for further exploration.

\section{SPECTRAL DENSITIES FROM MINIMAL STRINGS: DISC LEVEL}

The JT gravity (and the JT supergravity) partition function is structurally the same as a "macroscopic loop" expectation value in the old double-scaled matrix model language $[48,49]$, involving the trace of an effective onedimensional Hamiltonian [Eq. (3)] that arises naturally from the matrix model after double scaling:

$$
Z(\beta)=\int_{-\infty}^{\mu} d x\left\langle x\left|e^{-\beta \mathcal{H}(\hat{p}, \hat{x})}\right| x\right\rangle,
$$

where the upper limit on the $x$ integration, $\mu$, will be discussed below. Instead of fixed loop length $\ell$, the problem describes a fixed inverse temperature $\beta$, which is the length of the boundary of the nearly $\mathrm{AdS}_{2}$ spacetime here. As a quantum mechanics problem, to examine the disc-level physics, all that is needed is to work at leading order in $\hbar$. Denoting the leading/classical piece of the potential as $u_{0}(x)=\lim _{\hbar \rightarrow 0}(u(x))$, inserting a complete set of momentum states, and using the normalization $\langle x \mid p\rangle=$ $e^{i p x} / \sqrt{2 \pi \hbar}$ for the wave function, we write

$$
\begin{aligned}
Z_{0}(\beta) & =\int_{-\infty}^{\mu} d x \int_{-\infty}^{+\infty} \frac{d p}{2 \pi \hbar} e^{-\beta\left[p^{2}+u_{0}(x)\right]} \\
& =\frac{1}{2 \hbar \sqrt{\pi \beta}} \int_{-\infty}^{\mu} d x e^{-\beta u_{0}(x)} \\
& =\frac{1}{2 \pi \hbar} \sqrt{\frac{\pi}{\beta}} \int_{0}^{\infty} d u_{0} f\left(u_{0}\right) e^{-\beta u_{0}},
\end{aligned}
$$

where $f\left(u_{0}\right)=-\partial x / \partial u_{0}$. The fact that $u_{0}(\mu)=0$ was used, which will be confirmed below. The last integral can be written as

$$
\begin{aligned}
Z_{0}(\beta) & =\int_{0}^{\infty} d E \int_{0}^{E} \frac{f\left(u_{0}\right)}{\sqrt{E-u_{0}}} \frac{d u_{0}}{2 \pi \hbar} e^{-\beta E} \\
& =\int_{0}^{\infty} d E \rho_{0}(E) e^{-\beta E}
\end{aligned}
$$

where

$$
\rho_{0}(E)=\frac{1}{2 \pi \hbar} \int_{0}^{E} \frac{f\left(u_{0}\right)}{\sqrt{E-u_{0}}} d u_{0} .
$$

So, $\rho_{0}(E)$ is determined if $u_{0}(x)$ is known, since it defines the $f\left(u_{0}\right)$ kernel of the integral transform in Eq. (9). The lower limit is $u_{0}=0$ and can be seen to mark the $E=0$ end of the classical spectral density in this construction. A popular and important case is the simple ("Airy") potential, $u(x)=-x$. So $u_{0}(x)=-x$, and $f\left(u_{0}\right)=1$. This readily yields $\rho_{0}(E)=E^{1 / 2} / \pi \hbar$. This is the double-scaled limit of the famous Wigner semicircle law for a Gaussian Hermitian matrix model. 


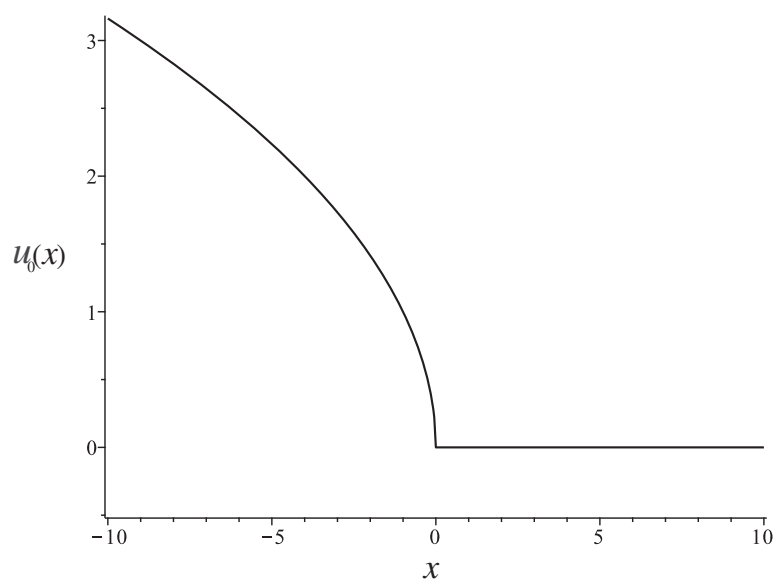

FIG. 2. Features of the leading potential for the minimal models. For $x<\mu, u_{0}(x)=(-x)^{1 / k}(k=2$ plotted here). This is common to both the bosonic and the type-0A minimal models. For $x>\mu, u_{0}(x)=0$, a key feature of the type-0A minimal models. [Fig. 5 shows the full $u(x)$ for $k=2$ type 0A.]

Of interest will be the behaviors indexed by integer $k$ : $u_{0}=(-x)^{1 / k}$ for large $x<0$, a well-known leading behavior for certain classes of minimal string models (i.e., the "multicritical" behavior in the old double-scaling language [50]). (See the left region of Fig. 2.) Simple scaling of Eq. (9) shows that this will yield a spectral density $\rho_{0}=C_{k} E^{k-\frac{1}{2}} / 2 \pi \hbar$, but the numerical coefficient $C_{k}$ will be important in what follows. A bit of work shows that, with $z=u_{0} / E$,

$$
\begin{aligned}
C_{k} & =k \int_{0}^{1} \frac{z^{k-1} d z}{\sqrt{1-z}}=2 k \int_{0}^{\pi / 2}(\sin \theta)^{2 k-1} d \theta \\
& =\frac{2^{2 k-1}((k-1) !)^{2} k}{(2 k-1) !},
\end{aligned}
$$

which can be proven by, e.g., expressing $(\sin \theta)^{n}$, for odd $n$, in terms of sums of terms involving $\sin (n \theta), \sin ((n-2) \theta)$, etc. The coefficients are sums of those in the binomial expansion.

Now, it is time to build more complicated spectral densities relevant to JT and super-JT. For any $k$, the potential $u_{0}=(-x)^{1 / k}$ increases as $x \rightarrow-\infty$, and so this form will dominate the large- $E$ behavior. A general minimal model (in this class) has the following defining equation for $u_{0}$, as $x \rightarrow-\infty$ :

$$
\sum_{k=1}^{\infty} t_{k} u_{0}^{k}=-x
$$

where $t_{k}$ is the coupling that turns on the $k$ th model. This equation is to be thought of as an interpolating flow connecting all the models. Such an equation is the leading piece of what was called a "string equation" in the older matrix model literature. (The full nonlinear string equations will be discussed in Sec. III.) So, in preparation for working with Eq. (9),

$$
f\left(u_{0}\right)=-\frac{\partial x}{\partial u_{0}}=\sum_{k=1}^{\infty} k t_{k} u_{0}^{k-1}
$$

The scheme to move forward with here is that any Schwarzian-type disc-level spectral density $\rho_{0}(E)$ that has a series expansion in powers of the form $E^{k-\frac{1}{2}}$ can be reconstructed from a potential $u_{0}(x)$ that can be deduced by using the ingredients above. It amounts to a specific formula for the $t_{k}$, determining the particular combination of minimal models to be used to build the JT gravity model. For example, in the case of the ordinary JT model, with the disc spectral density given in Eq. (2),

$$
\begin{aligned}
\rho_{0}(E) & =\frac{1}{4 \pi^{2} \hbar} \sum_{k=1}^{\infty} \frac{(2 \pi \sqrt{E})^{2 k-1}}{(2 k-1) !} \quad(\mathrm{JT}) \\
& =\frac{1}{4 \pi^{2} \hbar} \int_{0}^{E} \sum_{k=1}^{\infty} \frac{2^{2 k-1} \pi^{2 k-1}}{(2 k-1) !} \frac{k}{C_{k}} u_{0}^{k-1} \frac{d u_{0}}{\sqrt{E-u_{0}}} \\
& =\frac{1}{4 \pi \hbar} \int_{0}^{E} \sum_{k=1}^{\infty} \frac{\pi^{2 k-2} u_{0}^{k-1}}{(k-1) !^{2}} \frac{d u_{0}}{\sqrt{E-u_{0}}} \\
& =\frac{1}{4 \pi \hbar} \int_{0}^{E} \sum_{k=1}^{\infty} \frac{\left(\pi \sqrt{u_{0}}\right)^{2 k-2}}{(k-1) !^{2}} \frac{d u_{0}}{\sqrt{E-u_{0}}} \\
& =\frac{1}{2 \pi \hbar} \int_{0}^{E} \frac{I_{0}\left(2 \pi \sqrt{u_{0}}\right) d u_{0}}{2 \sqrt{E-u_{0}}}
\end{aligned}
$$

where $I_{0}(s)$ is the zeroth modified Bessel function of the first kind. From the above, it is clear from Eqs. (9) and (12) that the $t_{k}$ are

$$
t_{k}=\frac{\pi^{2 k-2}}{2 k !(k-1) !},
$$

as shown in Ref. [29] (with a different normalization), and used in Ref. [30] as described in the Introduction.

It is worth pausing to reflect on the meaning of this. First, and most importantly, note that the simple additive structure in Eq. (11) is deceptively simple. For each minimal model, the full string equation (see Sec. III) will be highly nonlinear in $u(x)$ and its derivatives, and the additive structure will couple together those nonlinear equations. So, the resulting solution $u(x)$ is not a simple sum of the behavior of the $u(x)$ 's from the individual models. Remarkably, however, $u(x)$ 's evolution as a function of the $t_{k}$ is described by the $k$ th (integrable) $\mathrm{KdV}$ flow equation [48,51], which takes the form

$$
\frac{\partial u}{\partial t_{k}} \propto \frac{\partial}{\partial x} \tilde{R}_{k+1}[u],
$$




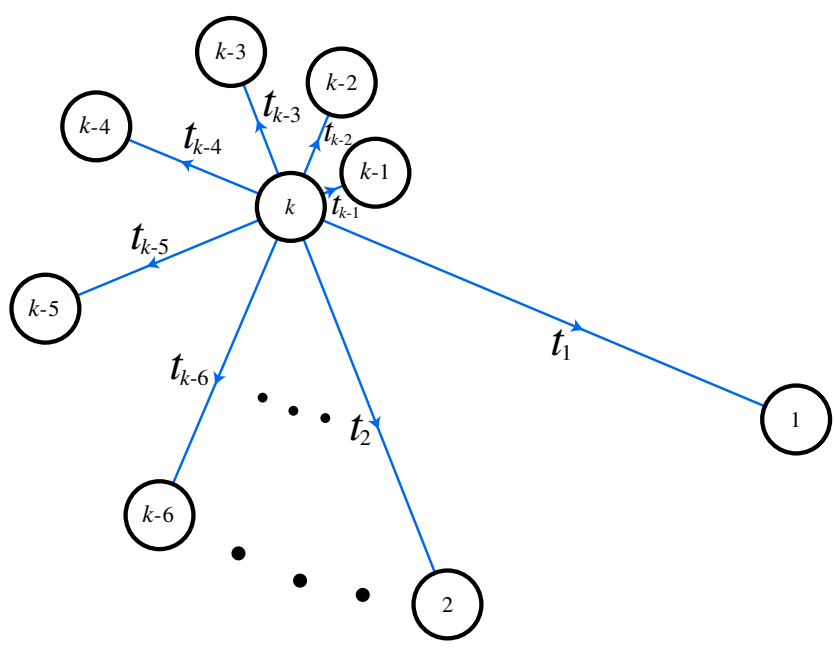

FIG. 3. A schematic diagram of the interpolating flow as a pattern of operator deformations. A circle with $i$ in it is the $i$ th minimal model. Here, $k$ should be understood to be taken to infinity. There are $k-1$ operators in the model, labeled $\mathcal{O}_{l}$, and deformation with coefficient $t_{l}$ is equivalent to turning on the $l$ th model. The lengths of the bonds/arrows signify the differing strengths of the $t_{l}$ 's.

where the $\tilde{R}_{k}$ 's are polynomials in $u(x)$ and its derivatives [examples are listed in Eq. (26)], and the $t_{k}$ 's are the times of the integrable flows. From a conformal field theory perspective, for, e.g., the bosonic $(2,2 k-1)$ minimal models (similar remarks hold for the type- $0 \mathrm{~A}$ models discussed later), the $k$ th model has $k-1$ operators, $\mathcal{O}_{l}$, $(l=1,2 \ldots, k-1)$, and deformation with $t_{l}$ is equivalent to turning on the operator $\mathcal{O}_{l}$. It is natural, therefore, to think of the result for the pattern of interpolating flows in Eq. (14) [and in Eq. (19) to be derived shortly for the supergravity in terms of type-0A minimal models] as simply a specific operator deformation, or RG flow from the (infinite) $k$ th model to the other models at lower $k$. (See Fig. 3 for a schematic diagram.) In this sense, the suggestions (on the one hand) of Ref. [17] that JT gravity is an infinite- $k$ limit of the $k$ th minimal model, and (on the other hand) of Ref. [29] that JT gravity (or supergravity, as discussed in this paper) is an infinite sum of all of them, are in fact complementary.

Next, it is important to consider low-energy physics, where the features of the potential $u(x)$ at intermediate $x$ and indeed $x>0$ become very important. In the case of minimal models derived from Hermitian matrix models, the leading potential $u_{0}(x)$ is of the form $x^{1 / k}$ as $x \rightarrow+\infty$, and this leads to nonperturbative problems at low energy. In fact, Ref. [30] used different minimal models that have the same large- $E$ physics as those minimal models, but with different, improved, low- $E$ physics. In those models, the leading-order low-energy physics of interest is simply $u_{0}(x)=0$ for all $k$, a striking universal feature. See Fig. 2. (This behavior is not arbitrarily imposed, but actually a solution of the underlying matrix model, as explained in Sec. III.) The feature of the spectral density that results from this can be seen by converting Eq. (9) back into an $x$ integral, viz.

$$
\rho_{0}(E)=\frac{1}{2 \pi \hbar} \int_{-\left|x_{0}\right|}^{\mu} \frac{d x}{\sqrt{E-u_{0}(x)}},
$$

(where the lower limit $x=-\left|x_{0}\right|$ is where the square root vanishes), and so the low-energy regime where $u_{0}(x)=0$ yields the leading behavior

$$
\rho_{0}(E)=\frac{1}{2 \pi \hbar} \int_{0}^{\mu} \frac{d x}{\sqrt{E}}=\frac{1}{2 \pi \hbar} \frac{\mu}{\sqrt{E}}+\cdots .
$$

The choice $\mu=2$ will be made here, a convention that reproduces the leading term of Eq. (4). Beyond low $E$, other physics takes over and the $\mu / \sqrt{E}$ behavior is modified, ultimately crossing over into behavior that is described by whichever combination of the $E^{k-\frac{1}{2}}$ physics appears at high $E$. In Ref. [30], that combination was chosen to be the same as the ordinary JT gravity model, with $\mu=0$, giving the same large- $E$ perturbative behavior as the JT matrix model of Ref. [17], but better, stable, nonperturbative physics at low $E$.

However, it is possible to instead reproduce the JT supergravity disc spectral density given in Eq. (4). That it is also a combination of minimal models (but with different coefficients) should follow from again expanding, and again using the integral representation [Eq. (9)] of positive half-integer powers of $E$. Doing so yields

$$
\begin{aligned}
\rho_{0}(E) & =\frac{1}{\pi \hbar \sqrt{E}}+\frac{2}{\hbar} \sum_{k=1}^{\infty} \frac{2^{2 k-1} \pi^{2 k-1}}{(2 k) !} E^{k-\frac{1}{2}} \quad(\mathrm{SJT}) \\
& =\frac{1}{\pi \hbar \sqrt{E}}+\frac{2}{\hbar} \int_{0}^{E} \sum_{k=1}^{\infty} \frac{2^{2 k-1} \pi^{2 k-1}}{(2 k) !} \frac{k u_{0}^{k-1}}{C_{k-1}} \frac{d u_{0}}{\sqrt{E-u_{0}}} \\
& =\frac{1}{\pi \hbar \sqrt{E}}+\frac{2 \pi}{\hbar} \int_{0}^{E} \sum_{k=0}^{\infty} \frac{\left(\pi \sqrt{u_{0}}\right)^{2 k}}{(2 k+2) k !^{2}} \frac{d u_{0}}{\sqrt{E-u_{0}}} \\
& =\frac{1}{\pi \hbar \sqrt{E}}+\frac{\pi}{\hbar} \int_{0}^{E} \frac{I_{1}\left(2 \pi \sqrt{u_{0}}\right)}{2 \pi \sqrt{u_{0}}} \frac{d u_{0}}{\sqrt{E-u_{0}}} .
\end{aligned}
$$

So remarkably, for the positive powers of $E$ in this supergravity case, a natural generalization of what was seen for the ordinary JT case emerges: $f\left(u_{0}\right)=\pi I_{1}\left(2 \pi \sqrt{u_{0}}\right) /$ $\left(2 \pi \sqrt{u_{0}}\right)$, where $I_{1}(s)$ is the first modified Bessel function of the first kind [cf. the last line in Eq. (13)]. The special $E^{-\frac{1}{2}}$ part of the spectral density will be reproduced if there is a leading contribution to the potential of form $u_{0}=0$, as discussed above. As already mentioned, the minimal models used below for the full (not just leading-order) potential will naturally have this behavior built in. So, this shows that the super-JT spectral density can be built out of a combination of minimal models with 


$$
t_{k}=\frac{\pi^{2 k-2}}{(k !)^{2}}
$$

A summary of the overall picture that contrasts the JT and SJT cases is as follows: Writing $y \equiv \pi \sqrt{E}$,

$$
\begin{aligned}
\text { SJT: } \hbar \rho_{0}(E) & =\frac{\cosh (2 y)}{y}=\frac{1}{y}+\frac{2 \sinh ^{2} y}{y} \\
& =\frac{1}{\pi \sqrt{E}}+\pi \int_{0}^{E} \frac{I_{1}\left(2 \pi \sqrt{u_{0}}\right)}{2 \pi \sqrt{u_{0}}} \frac{d u_{0}}{\sqrt{E-u_{0}}} . \\
\text { JT: } 4 \pi^{2} \hbar \rho_{0}(E) & =\sinh (2 y) \\
& =\pi \int_{0}^{E} I_{0}\left(2 \pi \sqrt{u_{0}}\right) \frac{d u_{0}}{\sqrt{E-u_{0}}} .
\end{aligned}
$$

The next step is to consider the perturbative physics beyond the disc and, of course, nonperturbative aspects.

\section{STRING EQUATIONS}

As stated before, in the approach to double-scaled matrix models that will mostly be taken here, the focus is on the potential $u(x)$ of the associated Hamiltonian problem [Eq. (3)]. In the minimal string approach of old, the nonlinear ODE that defines it was often called a "string equation." A sort of master string equation that contains all the behavior of current interest is $[42,44,45,52]$

$$
u \mathcal{R}^{2}-\frac{\hbar^{2}}{2} \mathcal{R} \mathcal{R}^{\prime \prime}+\frac{\hbar^{2}}{4}\left(\mathcal{R}^{\prime}\right)^{2}=\hbar^{2} \Gamma^{2},
$$

where for the $k$ th model,

$$
\mathcal{R} \equiv \tilde{R}_{k}[u]+x .
$$

Here, $\tilde{R}_{k}[u]$ is the $k$ th-order polynomial in $u(x)$ and its $x$ derivatives defined by Gel'fand and Dikii [53], but normalized so that the coefficient of $u^{k}$ is unity. For example,

$$
\begin{aligned}
\tilde{R}_{1}[u] & =u \\
\tilde{R}_{2}[u] & =u^{2}-\frac{1}{3} u^{\prime \prime}, \\
\tilde{R}_{3}[u] & =u^{3}-\frac{1}{2}\left(u^{\prime}\right)^{2}-u u^{\prime \prime}+\frac{1}{10} u^{\prime \prime \prime \prime}, \\
& \vdots \\
\tilde{R}_{k}[u] & =u^{k}+\cdots+\# u^{(2 k-2)} .
\end{aligned}
$$

Here a prime denotes an $x$ derivative times a factor of $\hbar$, and in the last line the superscript $(2 k-2)$ means that number of primes.

The string equation [Eq. (21)] was first derived (initially with $\Gamma=0$ ) by taking $[42,44,45]$ the double-scaling limit of models of a random complex matrix $M$, with polynomial potential $V\left(M^{\dagger} M\right)$. Diagonalization to work in terms of the positive quantities $\lambda_{i}^{2}$, where $\lambda_{i}$ are eigenvalues of $M$, shows that they are in the $(1,2)$ Altland-Zirnbauer class of matrix ensembles. It was soon realized [52] that nonzero $\Gamma$ could be introduced naturally from a number of different perspectives, including one where $\Gamma$ corresponds to having added $\Gamma$ quark flavors, or in modern language, $\Gamma$ background D-branes. There is an extra logarithmic term in the potential with coefficient $\Gamma$, which amounts to studying the $(2 \Gamma+1,2)$ class of matrix ensembles. Later, Ref. [47] supplied a type-0A interpretation, including an understanding of $\Gamma$ as also counting units of R-R flux.

In almost all the work in the literature on Eq. (21), integer $\Gamma$ was considered the most physical choice, although it was noticed that half-integer cases possessed certain interesting (but not fully explained) properties. (Reference [54] explored many of these properties, suggesting a partial physical understanding in terms of minimal string theories with no background D-branes.) In this paper, it will be made clear that solutions with half-integer $\Gamma$ are physical and play a very important role.

For orientation, the natural next step is to study some special cases.

\section{A. Bosonic minimal models}

Consider first the case of $\Gamma=0$. An obvious nontrivial solution to the string equation (21) is $\mathcal{R}=0$, defining a subset of equations for any $k$. These are simply the ODEs defining the original $(2,2 k-1)$ bosonic minimal models arising from double-scaling Hermitian matrix models [18-21]. The case $k=1$ is the Airy model $u(x)=-x$, the case $k=2$ is the Painlevé I equation defining pure gravity [with the leading $u_{0}=(-x)^{1 / 2}+\cdots$ ], and $k=3$ is the gravitating Lee-Yang model [with the leading $u_{0}=$ $\left.(-x)^{1 / 3}+\cdots\right]$, etc. Not much will be said about these beyond this point in this paper, but it is perhaps useful to signpost them for orientation.

\section{B. A universal model}

Another interesting special solution is the case where $\mathcal{R}=x$, in which case there is an exact solution Eq. (21):

$$
u(x)=0+\hbar^{2} \frac{\left(\Gamma^{2}-\frac{1}{4}\right)}{x^{2}} .
$$

So, the leading piece of the potential is $u_{0}=0$. This, as we have seen, will produce a $1 /(\pi \hbar \sqrt{E})$ behavior in the spectral density $\rho_{0}(E)$, and the order- $\hbar^{2}$ piece that comes next generates corrections. ${ }^{1}$ In fact, there is a nice way to organize

\footnotetext{
${ }^{1}$ This curious exact solution for $u(x)$, for $\Gamma=0$, was first noticed in Ref. [52] and referred to as the $k=0$ solution. It was generalized to an interesting infinite family of rational solutions in Ref. [54].
} 
the higher-order corrections, giving an opportunity to introduce a technique that will become useful later on.

\section{A resolvent method}

In fact, the spectral density can be written as

$$
\rho(E)=\frac{\operatorname{Im}}{\pi \hbar} \int_{a}^{b} \hat{R}(x, E) d x,
$$

where the quantity $\hat{R}(x, E) \equiv\left\langle x\left|(\mathcal{H}-E)^{-1}\right| x\right\rangle$ is the (diagonal of the) resolvent of the Schrödinger Hamiltonian $\mathcal{H}$ given in Eq. (3). The interval $[a, b]$ that is chosen will depend upon which $x$ regime is being studied, and will be discussed shortly. (The "Im" part of the prescription above means taking the imaginary part of the integrated resolvent as it approaches the real positive $E$ line.)

It is very important to note that this is not the resolvent expectation value $R(x)$ used in Refs. [17,33]. That is the resolvent of the raw random matrix of the matrix model, from which physics is subsequently extracted in the scaling limit. The resolvent here is that of the double-scaled Hamiltonian that arises after double scaling. The hat will hopefully go some way toward helping the reader separate the two objects when they consult those papers. The relation between them is that the $x$ integral (between appropriately chosen limits $a$ and $b$ ) of the resolvent of this paper is proportional to (the scaling part of) the resolvent of those papers.

Rather usefully, $\hat{R}(x, E)$ satisfies the Gel'fand-Dikii equation [53]:

$$
4(u-E) \hat{R}^{2}-2 \hbar^{2} \hat{R} \hat{R}^{\prime \prime}+\hbar^{2}\left(\hat{R}^{\prime}\right)^{2}=1,
$$

where $u=u(x)$, and a prime denotes a differentiation with respect to $x$. This equation supplies, for an input potential $u(x)$, the full (perturbative and nonperturbative) spectral density $\rho(E)$ via Eq. (25). This alternative approach for computing $\rho(E)$ is rather useful (see Ref. [55] for an earlier study in this type-0A context), and in fact Ref. [30] used the above equations to write a differential equation directly for $\rho(E, x)$. However, working directly with the resolvent is illuminating: Starting with $u(x)=u_{0}(x)$, the leading piece in the limit $\hbar=0$, all derivative terms can be dropped in Eq. (26), and the result [Eq. (16)] comes from the solution $\hat{R}=-1 /\left(2 \sqrt{u_{0}(x)-E}\right)$ (the sign is chosen to give a positive density). The case $u_{0}(x)=0$ in the integrand is between $x=0$ and $x=\mu$, giving the term $\hat{R}=-1 /(2 \sqrt{-E})$, and it leads to the contribution in Eq. (17). Expanding around this leading result gives, using Eq. (24), to order $\hbar^{2}$,

$$
\hat{R}=-\frac{1}{2 \sqrt{-E}}-\frac{\hbar^{2}\left(\Gamma^{2}-\frac{1}{4}\right)}{4 x^{2}(-E)^{3 / 2}}+\cdots,
$$

which yields, using Eq. (25),

$$
\rho(E)=\frac{1}{\pi \hbar \sqrt{E}}-\frac{1}{8}\left(\Gamma^{2}-\frac{1}{4}\right) \frac{\hbar}{\pi E^{3 / 2}}+\cdots
$$

The second term should be thought of as a combination of $\rho_{1,0}(E)$ (genus 1 with one boundary) and $\rho_{0,2}$ (one boundary and two crosscaps), depending upon the interpretation of $\Gamma$.

Of course, there are nonperturbative contributions to $\rho(E)$ that cannot be obtained by this perturbative procedure. It was shown in Ref. [56] that the wave function $\psi(E, x)$ of this particular Hamiltonian problem [defined by potential in Eq. (24)] can be written in closed form in terms of Bessel functions of the first kind, $J_{\Gamma}$ :

$$
\psi(E, x)=\frac{1}{\sqrt{2} \hbar} x^{\frac{1}{2}} J_{\Gamma}\left(\frac{\sqrt{E} x}{\hbar}\right),
$$

and the spectral density can be computed $[30,57,58]$ :

$$
\begin{aligned}
\rho_{J}(E, \mu) & =\int_{0}^{\mu}|\psi(E, x)|^{2} d x=\frac{1}{4 E} \int_{0}^{\frac{E \mu^{2}}{\hbar^{2}}} J_{\Gamma}^{2}(\sqrt{t}) d t \\
& =\frac{\mu^{2}}{4 \hbar^{2}}\left[J_{\Gamma}^{2}(\xi)+J_{\Gamma+1}^{2}(\xi)-\frac{2 \Gamma}{\xi} J_{\Gamma}(\xi) J_{\Gamma+1}(\xi)\right],
\end{aligned}
$$

where $\xi \equiv \mu \sqrt{E} / \hbar$.

Expanding this result with $\mu=2$ yields the previously obtained perturbative terms, as well as nonperturbative terms oscillatory in $\sqrt{E} / \hbar$.

This "Bessel" model is important, since it furnishes an exact model of the low-energy sector for all the minimal models, and for the full JT supergravity. For the special cases of $\Gamma= \pm \frac{1}{2}$ [corresponding to the special $(0,2)$ and $(2,2)$ cases discussed in Ref. [33]], things become extremely simple. Since ordinary Bessel functions of halfinteger order can be written in terms of trigonometric functions, specifically for this case,

$$
J_{\frac{1}{2}}(\xi)=\sqrt{\frac{2}{\pi \xi}} \sin \xi, \quad \text { and } \quad J_{-\frac{1}{2}}(\xi)=\sqrt{\frac{2}{\pi \xi}} \cos \xi,
$$

and using $J_{\frac{3}{2}}(\xi)=\xi^{-1} J_{\frac{1}{2}}(\xi)-J_{-\frac{1}{2}}(\xi)$, the asymptotic form given in Eq. (28) truncates to the leading piece, and remarkably the full perturbative and nonperturbative parts are contained in this compact form:

$$
\rho_{ \pm \frac{1}{2}}=\frac{1}{\pi \hbar \sqrt{E}} \mp \frac{1}{4 \pi E} \sin \left(\frac{4 \sqrt{E}}{\hbar}\right) .
$$

These cases are plotted in Fig. 4.

The $E \rightarrow 0$ behaviors of these two cases are markedly different. For $\Gamma=+\frac{1}{2}$, in the limit there is a nice cancellation between the first and second terms, and so $\rho_{\frac{1}{2}} \rightarrow 0$. 


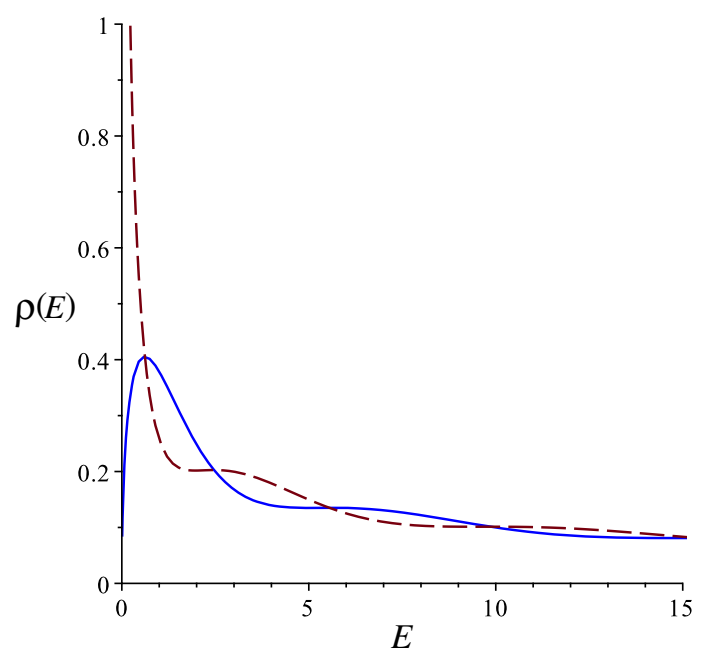

FIG. 4. The special Bessel spectral densities for $\Gamma=\frac{1}{2}$ (solid) and $\Gamma=-\frac{1}{2}$ (dashed).

There is a doubling instead of a cancellation for $\Gamma=-\frac{1}{2}$, resulting in an $E^{-1 / 2}$ divergence for $\rho_{-\frac{1}{2}}$, even after nonperturbative effects are taken into account. These two behaviors will be seen again in the full nonperturbative definition building JT supergravity out of minimal models, proposed and explored in Sec. IV.

From the point of view of the recursive construction of Stanford and Witten [33], the vanishing of all orders in perturbation theory (beyond the disc for the spectral density, or the disc + crosscap for the accompanying resolvent) is a sort of perturbative miracle. ${ }^{2}$ Here, working directly with the potential $u(x)$, it is easier to isolate its origins, and to anticipate what can happen in the full model defined in the next section. Looking at the resolvent's differential equation [Eq. (26)], it is clear that exact vanishing of the potential at $\Gamma= \pm \frac{1}{2}$ guarantees no perturbative corrections to $\hat{R}(E, x)$ [and hence $\rho(E)$ ] beyond the disc. While the potential vanishes, there is still, however, a nontrivial equation for $\hat{R}(E, x)$, and this will encapsulate the nonperturbative physics. A nice way to write it is to take another derivative. The structure of the equation is such that there is a cancellation between terms, and an overall factor of $\hat{R}$ can be divided out, and for vanishing $u$,

$$
\hat{R}^{\prime \prime \prime}=-4 E \hat{R}^{\prime}
$$

with an obvious solution of a constant [already found to be $-1 /(2 \sqrt{-E})]$ plus an oscillatory piece with frequency $2 \sqrt{E} / \hbar$. A natural choice of its coefficient is to make the resolvent vanish at $x=0$, yielding

\footnotetext{
${ }^{2}$ Also, it accompanies the fact that in this special case, the whole perturbative analysis is saved from being afflicted by a divergence in the volume of the one-crosscap moduli space that would propagate to higher orders through recursion relations.
}

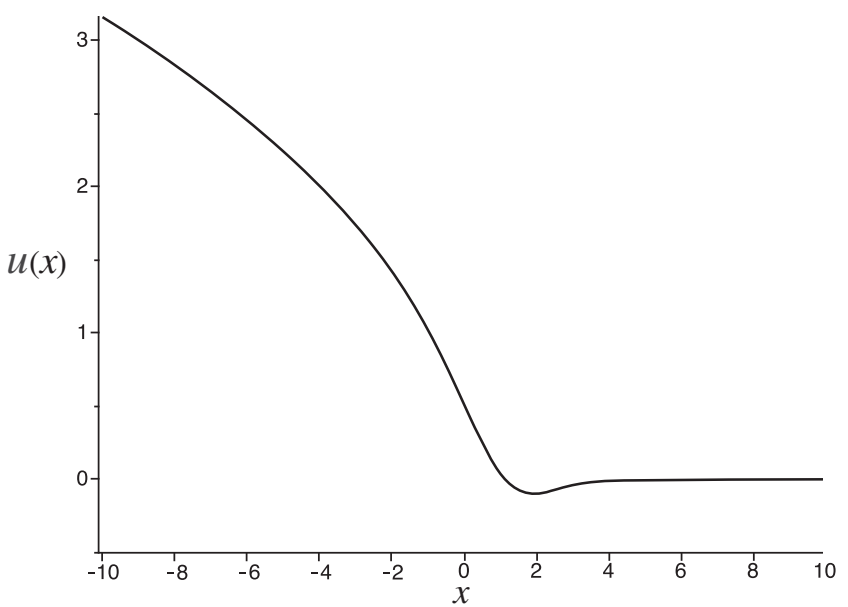

FIG. 5. The potential $u(x)$ that is supplied by Eq. (21) for the case $k=2$. Cf. Fig. 2 for the leading part, $u_{0}(x)$, in this case.

$$
\hat{R}(E, x)=-\frac{1}{2 \sqrt{-E}}\left[1-\exp \left(i \frac{2 \sqrt{E}}{\hbar} x\right)\right] .
$$

Integrating this between $x=0$ and $x=\mu=2$ gives an imaginary piece, which [via Eq. (25)] gives the exact density in Eq. (32) for $\Gamma=+\frac{1}{2}$. (The case $\Gamma=-\frac{1}{2}$ giving the relative minus sign does not seem as natural here.)

Crucially, there is also a nonoscillatory term, $1 /(4 \pi E)$, coming from the upper limit of the integration of the exponential. It has the interpretation as a crosscap contribution, but it does not appear in the density, since it is real. This is the finite crosscap term discussed in Ref. [33] in this $\Gamma=\frac{1}{2}$ case (and its $\Gamma=-\frac{1}{2}$ counterpart).

\section{Type-0A minimal models}

As should be clear from the previous section, the potentials that are needed to construct JT supergravity are ones which contain both types of behavior-where, for nonzero $\Gamma$,

$u(x)=(-x)^{\frac{1}{k}}+\frac{\hbar \Gamma}{k(-x)^{1-\frac{1}{2 k}}}+\cdots \quad x \rightarrow-\infty$,
$u(x)=0+\frac{\hbar^{2}\left(\Gamma^{2}-\frac{1}{4}\right)}{x^{2}}+\cdots \quad x \rightarrow+\infty$.

For $\Gamma=0$, the large negative- $x$ behavior is identical to that of the bosonic minimal models, derived from Hermitian matrix models. This is what inspired their original study $[42,44,45]$ as alternative formulations of minimal string theories that had the same perturbative physics as the bosonic case, but better nonperturbative behavior. This also motivated a definition [30] of nonperturbative JT gravity with them based on the choice of $t_{k}$ that yields the disc spectral density [Eq. (2)] at large $E$. Note that the leading large positive- $x$ behavior is $k$-independent, showing a kind of universality. Figure 5 shows the $k=2$ example, constructed numerically, of one of these solutions. [Compare it to the leading piece, $u_{0}(x)$, shown in Fig. 2.] 


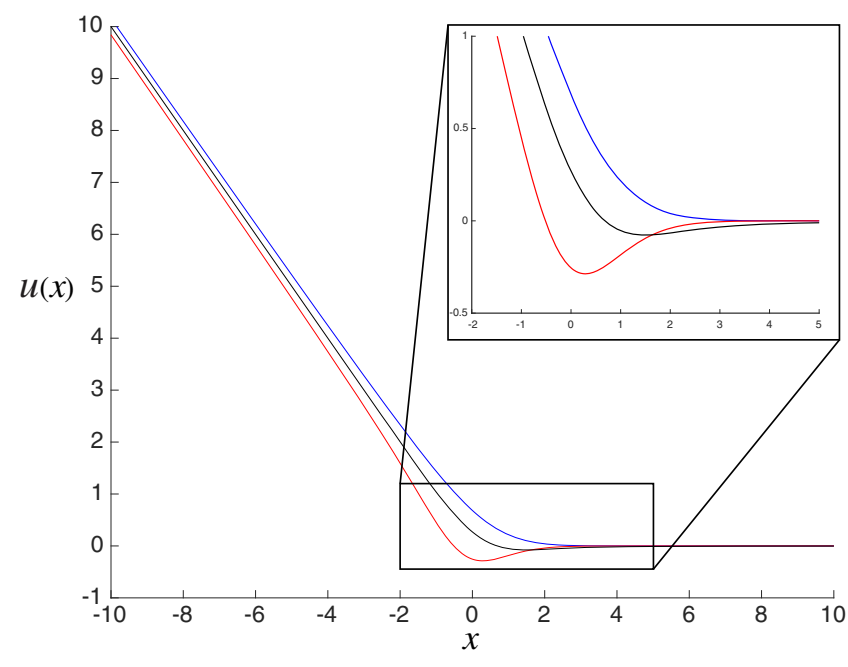

FIG. 6. The (mostly) central curve is the unique $(k=1) \Gamma=0$ solution to a special equation [Eq. (21)] derived from a matrix model. Also shown are the $k=1$ solutions for the special cases $\Gamma=\frac{1}{2}$ (uppermost) and $\Gamma=-\frac{1}{2}$ (lowermost).

The $k$ th model is in fact the $(2,4 k)$ type-0A minimal string theory: For nonzero $\Gamma$, in any given minimal model, the first subleading term in the large negative- $x$ regime has the interpretation [where two integrations of $u(x)$ gives the minimal string free energy] as a disc term, with $\Gamma$ counting the Chan-Paton labels for open string sectors, i.e., D-branes [52]. Also, in the minimal string picture, the large positive$x$ regime is a purely closed string expansion $[44,45]$ with the interpretation [47] that $\Gamma$ counts R-R flux insertions in (type-0A) string theory. In the large positive- $x$ regime, there are features of the perturbative expansion that are common to all $k$, and these will govern key aspects of the JT gravity defined by combining them, as will become clear.

These types of solutions have been studied a lot, starting with Refs. [42,44-46,52], and more recently Ref. [59]. There is strong evidence that there is a unique, smooth solution for $u(x)$ with those asymptotics for each $k$. This is proven for $k=1$ (with $\Gamma=0$ ), since in that case there is a map from the string equation to the Painleve II equation, for which the relevant solution was constructed by Hastings and McLeod [60]. A generalization of their methods was applied to the wider class of solutions in Ref. [61], providing strong evidence for their existence. Moreover, the map established in Ref. [52] between its solutions and those of the Painlevé II hierarchy suggests uniqueness for some nonzero $\Gamma$ via the results of Refs. [62,63]. Moreover, the underlying KdV flow structure (see Sec. II), which can evolve the unique $k=1$ solution into solutions for other $k$, suggests that they exist, if not on their own ensuring smoothness. More recently, a 't Hooft-like large- $\Gamma$ limit of the string equation was discussed in Ref. [47], which also suggests smooth solutions exist for all $k$. Numerical studies started back in Ref. [44], and since then the solutions have been exhibited numerically for numerous $k$ (and $\Gamma$ ). See
Fig. 6 for the $k=1$ case, with the curves for $\Gamma=0$ and $\Gamma= \pm \frac{1}{2}$ superimposed.

\section{JT SUPERGRAVITY FROM MINIMAL MODELS}

It is easy to define a general interpolating type- $0 \mathrm{~A}$ model $^{3}$ as well, simply by using the string equation (21) with asymptotics [Eq. (35)], and as input,

$$
\mathcal{R}=\sum_{k=1}^{\infty} t_{k} \tilde{R}_{k}[u]+x,
$$

with the specific choice of $t_{k}$ given in Eq. (19), ensuring that the leading part of $u(x)$ will yield the disc contribution that satisfies Eq. (11), and the disc spectral density will be that of the SJT model. The full string equation then gives the perturbative corrections to the disc, and the nonperturbative physics beyond. In short, this is the fully nonperturbative definition of the family of JT supergravity models.

The next two sections will examine perturbative features of this definition, coming from the two regimes where the potential (see, e.g., Figs. 5 and 6) is perturbative: large positive $x$ and large negative $x$. Equivalently, and perhaps more physically, these can alternatively be thought of as regimes where $\hbar$ is small, with $x$ either positive or negative. Perturbation theory is then organized in terms of powers of $\hbar$. Perturbative physics from positive $x$ and negative $x$ are separate families of corrections that should not be mixed together, and there is a choice as to which family should be used for expanding around the leading disc contribution given by $u_{0}(x)$. The JT supergravity definition has the spectral density determined by the integral (6), with $\mu$ positive (equal to 2 in our conventions, as already discussed). Small $\hbar$ is to be understood as small compared in magnitude to $\mu$, which is in the $x>0$ regime. So, positive $x$ is therefore the meaningful perturbative regime. (Conversely, were $\mu$ chosen to be negative, the meaningful perturbative expansion to develop would be from the $x<0$ regime, but that is not the situation here. $)^{4}$

So, perturbative corrections away from the $\rho_{0}(E)=$ $\cosh (2 \pi \sqrt{E}) / \pi \hbar \sqrt{E}$ already determined from the $u_{0}(x)$ will come from expanding $u_{0}(x)$ around 0 (its leading value

\footnotetext{
${ }^{3}$ Reference [61] studied, in the context of (type-0A) minimal strings, interpolations of this sort, following similar work on the bosonic minimal models in Ref. [64].

${ }^{4}$ The case $\mu=0$ is special, as it resembles the choice usually made for ordinary JT gravity. To really connect to ordinary JT gravity, it should be understood as $\mu=0^{-}$, thereby invoking the $x<0$ perturbation theory that coincides with the Hermitian matrix models for which the string equation is simply $\mathcal{R}=0$. This is, in fact, what is used in Ref. [30], to use these type-0A minimal models [with $t_{k}$ choice (14)] to give a nonperturbative definition of JT gravity that coincides perturbatively with that given by Hermitian matrix models.
} 
for $x>0$ ) in the positive- $x$ regime, and using the resolvent Eq. (26) to develop an expansion for $\hat{R}(x, E)$, and finally using Eq. (25) with limits $[a, b]=[0, \mu]$ (where $\mu=2$ in this paper's conventions).

\section{A. Perturbation theory: Positive $x$}

A most important feature of the definition is the fact that in the positive- $x$ regime, the potential has the perturbative form $u(x)=\hbar^{2}\left(\Gamma^{2}-\frac{1}{4}\right) / x^{2}+\cdots$. This controls the structure of the leading low-energy behavior, as already discussed above. The exact model of this sector, discussed in Secs. III B and III C, has this leading behavior. But the behavior beyond this is different for each $k$. So they supply an infinite family of generalizations of the model, but with one important feature: The factor $\left(\Gamma^{2}-\frac{1}{4}\right)$ multiplies the entire asymptotic series (for large positive $x$ ) for any $k$. For example, for $k=1$,

$$
\begin{aligned}
u(x)= & \frac{\left(\Gamma^{2}-\frac{1}{4}\right) \hbar^{2}}{x^{2}}-2 \frac{\left(\Gamma^{2}-\frac{1}{4}\right)\left(\Gamma^{2}-\frac{9}{4}\right) \hbar^{4}}{x^{5}} \\
& +7 \frac{\left(\Gamma^{2}-\frac{1}{4}\right)\left(\Gamma^{2}-\frac{9}{4}\right)\left(\Gamma^{2}-\frac{21}{4}\right) \hbar^{6}}{x^{8}}+\cdots,
\end{aligned}
$$

and similarly, for $k=2$,

$$
\begin{aligned}
u(x)= & \frac{\left(\Gamma^{2}-\frac{1}{4}\right) \hbar^{2}}{x^{2}}-2 \frac{\left(\Gamma^{2}-\frac{1}{4}\right)\left(\Gamma^{2}-\frac{9}{4}\right)\left(\Gamma^{2}-\frac{25}{4}\right) \hbar^{6}}{x^{7}} \\
& +\frac{11}{48}\left(\Gamma^{2}-\frac{1}{4}\right)\left(\Gamma^{2}-\frac{9}{4}\right)\left(\Gamma^{2}-\frac{25}{4}\right) \\
& \times \frac{\left(48 \Gamma^{4}-1240 \Gamma^{2}+8371\right) \hbar^{10}}{x^{12}}+\cdots .
\end{aligned}
$$

From here it is possible to work out $\hat{R}(x, E)$, and hence the contributions this sector makes to the density $\rho(E)$ beyond the orders given in Eq. (28), using the resolvent techniques of Sec. III C. For example, for $k=1$, the next order is

$$
\begin{aligned}
& \rho_{2,0}(E)+\rho_{1,2}(E)+\rho_{0,4}(E) \\
& =-\frac{\hbar^{3}}{128 \pi}\left(\Gamma^{2}-\frac{1}{4}\right)\left(\Gamma^{2}-\frac{9}{4}\right) \frac{(1-E)}{E^{5 / 2}},
\end{aligned}
$$

while for $k=2$, it is almost the same at this order [but with the $(1-E)$ in brackets replaced by $\frac{1}{2}$ ], followed by

$$
\begin{aligned}
& \rho_{4,0}(E)+\rho_{2,2}(E)+\rho_{1,4}(E)+\rho_{0,6}(E) \\
& =-\frac{\hbar^{5}}{(32)^{2} \pi}\left(\Gamma^{2}-\frac{1}{4}\right)\left(\Gamma^{2}-\frac{9}{4}\right)\left(\Gamma^{2}-\frac{25}{4}\right) \frac{\left(1-\frac{4}{3} E^{2}\right)}{E^{7 / 2}}
\end{aligned}
$$

at the next order.

Several remarks are due at this point. Perhaps the most important is that for $\Gamma= \pm \frac{1}{2}$, the entire perturbative series beyond the disc order $\rho_{0,0}$ vanishes - for all the general $k$ models from which the model is built-as happens in the special case of the exact Bessel model of Sec. III C, and as anticipated in Stanford and Witten's general perturbative analysis [33] for the full $(0,2)$ and $(2,2)$ supergravities. This is highly suggestive that this prescription for building JT supergravity from these minimal models is correct.

Indeed, this feature is definitely present in the full interpolating theory that is proposed for the complete interpolating definition. This follows from the special nature of the equation in this regime. The whole perturbative solution of Eq. (21), regardless of the form of the input $\mathcal{R}$ (interpolating or not), is seeded by the $k$-independent leading solution $u=\hbar^{2}\left(\Gamma^{2}-\frac{1}{4}\right) / x^{2}$. As a demonstration, we pick the interpolating case

$\mathcal{R}=t_{2} \tilde{R}_{2}+t_{1} \tilde{R}_{1}+x=t_{2}\left(-\frac{1}{3} u^{\prime \prime}+u^{2}\right)+t_{1} u+x$.

Working perturbatively, the solution leads with the universal piece, followed by the $k=1$ behavior, with $k=2$ behavior appearing at the next order, then both appearing mixed in the expected nonlinear fashion:

$$
\begin{aligned}
u(x)= & \left(\Gamma^{2}-\frac{1}{4}\right)\left\{\frac{\hbar^{2}}{x^{2}}+\left(\Gamma^{2}-\frac{9}{4}\right)\left\{-2 \frac{t_{1} \hbar^{4}}{x^{5}}\right.\right. \\
& +\frac{\hbar^{6}}{x^{8}}\left[7 t_{1}^{2}\left(\Gamma^{2}-\frac{21}{4}\right)-2 t_{2} x\left(\Gamma^{2}-\frac{25}{4}\right)\right] \\
& \left.\left.+\frac{\hbar^{8}}{x^{11}}\left[t_{1}^{3}\left(\operatorname{poly}_{1}\right)+t_{1} t_{2}\left(\operatorname{poly}_{2}\right)\right]\right\}+\cdots\right\},
\end{aligned}
$$

where poly $y_{1,2}$ are fourth-order polynomials in $\Gamma$. The key feature is the factor of $\Gamma^{2}-\frac{1}{4}$. This has an obvious generalization to the fully interpolating model. So, the vanishing of all orders at $\Gamma= \pm \frac{1}{2}$ is guaranteed in the full proposed JT supergravity in this perturbative regime.

Another remark can be made about the (probably already evident) intriguing pattern that is emerging. As $k$ increases, there are further special points at half-integer $\Gamma$. In such cases, the entire series for $u(x)$ truncates to the leading $C \hbar^{2} / \bar{x}^{2}$ term for some constant $C$. For example, $k=1$ has the case $\Gamma= \pm \frac{3}{2}$, for which $u(x)=2 \hbar^{2} / \bar{x}^{2}$ exactly. The $k=2$ model also has that case, and in addition $\Gamma= \pm \frac{5}{2}$, for which $u(x)=6 \hbar^{2} / \bar{x}^{2}$ exactly, and so on to higher $k$. Each of these has their exact spectral density at low energy given by Eq. (30), with the physics at all energies furnished by the complete string equation (21). There is again a truncation of the perturbative series to a finite number of terms, and all the nonperturbative physics can be written in terms of factors of oscillatory pieces with frequency $2 \sqrt{E} / \hbar$. This follows from the fact that, just as before, Bessel functions of half-integer order can be written as combinations of trigonometric functions. 
For example, working out the $\Gamma=\frac{3}{2}$ case explicitly is interesting. The perturbative contributions are the disc and the term that would previously be interpreted as the disc + handle added to the disc and two crosscaps, given by Eq. (28) with $\Gamma=\frac{3}{2}$ inserted. Once again, there are real terms in $\int_{0}^{2} \hat{R}(x, E) d x$ coming from the lower limit that correspond to crosscap order, as happened for $\Gamma=\frac{1}{2}$. However, in this case they are divergent. It is natural to suggest that this corresponds to the crosscap divergence seen by Stanford and Witten for $\Gamma \neq \pm \frac{1}{2}$, but this should be further explored. Nevertheless, the density, which seems to involve only even numbers of crosscaps, is finite and gives a well-behaved function, presumably defining a sensible theory for these broader values of $\Gamma$.

The presence of other special points for half-integer $\Gamma$ giving $u \sim \hbar^{2} / \bar{x}^{2}$ suggests further special circumstances in the theory of JT supergravity. In fact, there is a much richer story, which was elucidated in Ref. [54]. There, it was shown that at half-integer $\Gamma$ there are many rational solutions for $u(x)$ for each $k$, in the form of a ratio of polynomials in $x$ differing by 2 orders. This gives an expression for $u(x)$ that starts as $C \hbar^{2} / \bar{x}^{2}+\cdots$ for some constant $C$ and then truncates at some order.

\section{B. Perturbation theory: Negative $x$}

As already mentioned above, perturbation corrections beyond $\rho_{0}(E)$ come from the expansion in the positive- $x$ regime discussed in the previous section. There, it was confirmed that certain special features of JT supergravity observed by Stanford and Witten are naturally reproduced by the special properties of the string equation in this regime. However, it is interesting to explore the structure of the negative- $x$ expansion. (This would be physically relevant for the case of $\mu=0^{-}$; for example, relevant to ordinary JT gravity were $\Gamma=0$; see footnote 4 .) Moreover, it will uncover a useful piece of physics missing from the perturbative description of the previous section.

For each $k$, the leading form is given in the first line of Eq. (35). As already discussed, the first (classical) piece generates the contribution to the disc order that rises as $E^{k-\frac{1}{2}}$, for each $k$. In this regime, there is no universal leading form for $u(x)$ across all models, and so general statements about the nature of the potential in the interpolating model are harder to make. It is useful, however, to look at some of the behavior at individual $k$ values, and the case of $k=1$ is a good starting point. Going to a few orders beyond the leading ones, the potential is

$$
\begin{aligned}
u(x)=-x & \pm \frac{\Gamma \hbar}{(-x)^{1 / 2}}-\frac{1}{2} \frac{\Gamma^{2} \hbar^{2}}{x^{2}} \pm \frac{5}{32} \frac{\Gamma\left(4 \Gamma^{2}+1\right) \hbar^{3}}{(-x)^{7 / 2}} \\
& +\frac{1}{8} \frac{\Gamma^{2}\left(8 \Gamma^{2}+7\right) \hbar^{4}}{x^{5}} \cdots .
\end{aligned}
$$

Just as before, the resolvent technique of Sec. III C can be used to study the resulting spectral density. Some care must be taken. Here, the integral over $x$ is from $-\infty$ to 0 , and as before, finite physics can come from either limit. As a test of this methodology, consider the case $\Gamma=0$. Then, the expansion of the resolvent is entirely generated from the leading $u(x)=-x$ term, and the result for the density should coincide with the result (expanded) of the Airy model. Indeed, expanding Eq. (26) gives

$$
\begin{aligned}
\hat{R}(x, E)_{\Gamma=0}= & -\frac{1}{2 \sqrt{-(x+E)}}-\frac{5}{64} \frac{\hbar^{2}}{(-(x+E))^{7 / 2}} \\
& -\frac{1155}{4096} \frac{\hbar^{4}}{(-(x+E))^{13 / 2}}+\cdots,
\end{aligned}
$$

and so integrating and using Eq. (25) gives

$$
\rho(E)_{\Gamma=0}=\frac{\sqrt{E}}{\pi \hbar}+\frac{1}{32 \pi} \frac{\hbar}{E^{5 / 2}}-\frac{105}{2048 \pi} \frac{\hbar^{3}}{E^{11 / 2}}+\cdots,
$$

which is indeed the first few terms of the large- $E$ expansion of the exact expression for spectral density of the Airy model:

$\rho_{\mathrm{Ai}}(E)=\hbar^{-2 / 3}\left(\operatorname{Ai}^{\prime}(\zeta)^{2}-\zeta \operatorname{Ai}(\zeta)^{2}\right), \quad \zeta \equiv-\hbar^{-2 / 3} E$.

The first term in Eq. (45) is the disc, accompanying the $1 /(\pi \sqrt{E})$ present in Eq. (28), the next has a handle added, then two handles, and so forth.

Having done that, consider $\Gamma \neq 0$. Integrating the expanded resolvent gives, at the first two nontrivial orders with nonzero $\Gamma$,

$$
\left[-\frac{1}{2} \frac{ \pm \Gamma \hbar \sqrt{-x}}{E \sqrt{-(x+E)}}-\frac{1}{32} \frac{4 \Gamma^{2} \hbar^{2}}{(-(x+E))^{5 / 2}}\right]_{-\infty}^{0},
$$

where a term that is either zero or divergent at the limits has been neglected. The first term produces a crosscap term $\pm \hbar \Gamma / 2 E$ from the lower limit, and zero from the upper limit; and the second term produces an imaginary piece from the upper limit which produces a double crosscap contribution to the density, and zero from the lower limit. Checking a few more orders in this manner completes the expanded Airy result [Eq. (45)] for the density to

$$
\begin{aligned}
\rho(E)= & \frac{\sqrt{E}}{\pi \hbar}+\frac{\left(4 \Gamma^{2}+1\right)}{32 \pi} \frac{\hbar}{E^{5 / 2}} \\
& -\frac{\left(336 \Gamma^{4}+664 \Gamma^{2}+105\right)}{2048 \pi} \frac{\hbar^{3}}{E^{11 / 2}}+\cdots .
\end{aligned}
$$

In fact, the crosscap term must be treated with care, and it produces a special contribution. ${ }^{5}$ A single pole, $1 / E$, produces an imaginary part discontinuity that is a delta function: $1 /(E-i \epsilon)-1 /(E+i \epsilon)=2 i E /\left(E^{2}+\epsilon^{2}\right) \rightarrow 2 \pi i \delta(E)$,

\footnotetext{
${ }^{5}$ The author thanks Felipe Rosso for pointing out this term's contribution.
} 
and so there is a delta function contribution to the density from $\Gamma$ states at zero energy: $\rho(E)_{\text {crosscap }}= \pm \Gamma \delta(E)$. An analogous term arises for any $k$, and presumably for the full interpolating model, but it is the $k=1$ model that will dominate the low-energy physics of the interpolating model.

Such a contribution, and its significance, was discussed in Ref. [33] [by Laplace transform, it corresponds to adding $|\Gamma|$ to the partition function $Z(\beta)]$. Even though it was found in the "wrong" perturbative regime here, it is relevant to JT supergravity. Such a term is invisible in $x>0$ perturbation theory and should be accessible only using a nonperturbative approach. In a sense, the $x<0$ perturbation theory of this section (relevant to a negative- $\mu$ theory) acts as a nonperturbative probe of the model from the point of view of the positive $\mu$ case in hand. The result can be trusted because it is $\mu$-independent.

\section{Nonperturbative results}

Now it is time to turn to nonperturbative features of these type- $0 \mathrm{~A}$ minimal models, including new ones that go beyond the special features of the exact Bessel model of Sec. III C. They will act as toy models of the nonperturbative features of the full JT supergravity.

While it is not possible to write down the complete spectral density for the fully interpolating minimal models (since the string equation becomes formally of infinite order), many of the key features are clear from looking at any particular $k$. For $k=1$, for example, the spectral densities for the cases $\Gamma= \pm \frac{1}{2}$ were computed numerically using the same techniques employed ${ }^{6}$ for $\Gamma=0$ in Ref. [30], and they are presented in Figs. 7 and 8. To the left, there is the $1 / \sqrt{E}$ behavior at the disc level, plus nonperturbative corrections, and just as in the special $\Gamma=$ $\pm \frac{1}{2}$ models studied exactly in Sec. IIIC (see Fig. 4), for $\Gamma=+\frac{1}{2}$, nonperturbative effects cancel the divergence to zero, while for $\Gamma=-\frac{1}{2}$, the divergence is enhanced. Rather than falling off at larger $E$ (as the special Bessel models do), in this case new physics turns on and generates the rise to the right, attaining the $E^{\frac{1}{2}}$ disc asymptote at large $E$. A final important nonperturbative feature is, of course, the oscillatory modulation, indicative of the underlying random matrix model structure, showing the effects of repulsion of the eigenvalues. The basic frequency is $2 \sqrt{E} / \hbar$ [universal for all $k$, and hence for the full JT supergravity, because of the structure of the resolvent Eq. (26)], increasing to the right, but the amplitude is also suppressed at larger $E$.

Note that these features are present for any $k$ and the full interpolating model defining the JT supergravity. (Of

\footnotetext{
${ }^{6}$ Using a matrix Numerov method [65], the Schrödinger problem of Eq. (3) was solved with $-100 \leq x \leq+100$ on a grid of $4000 \times 4000$. A suitable normalization was performed for the 4000 eigenfunctions, and then the spectral density was constructed using a simple trapezoidal integration. See Ref. [30] for details.
}

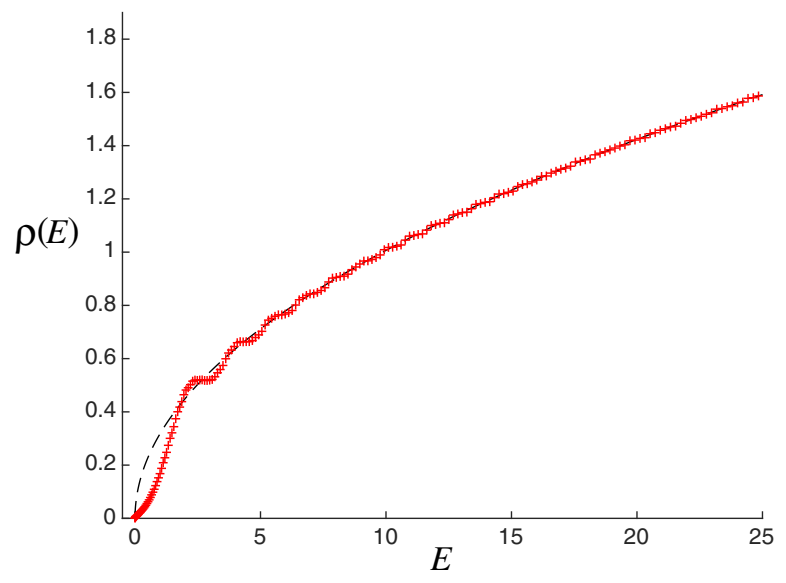

FIG. 7. The $k=1$ spectral density for $\Gamma=\frac{1}{2}$.

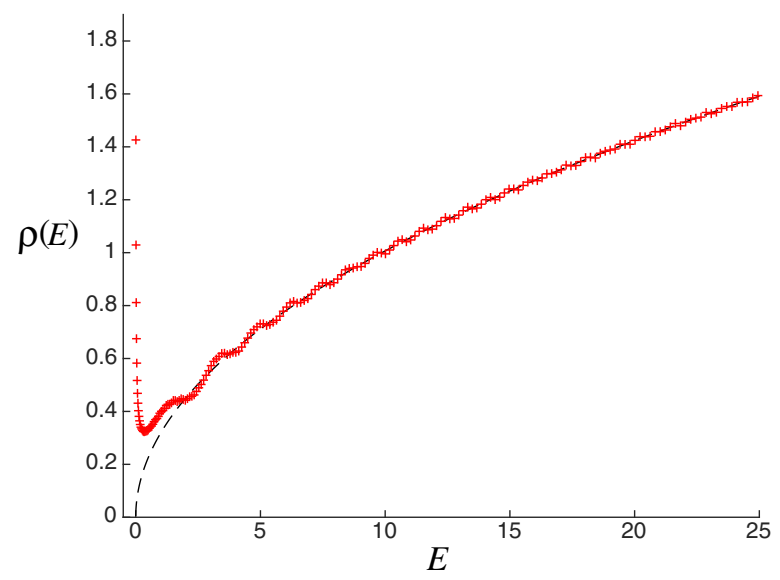

FIG. 8. The $k=1$ spectral density for $\Gamma=-\frac{1}{2}$.

course, there the rise with $E$ is not $E^{k-\frac{1}{2}}$ as for the $k$ th model, but rather exponential.)

Finally, the nonperturbative spectral density for other half-integer $\Gamma$ cases can be readily computed. Again, for

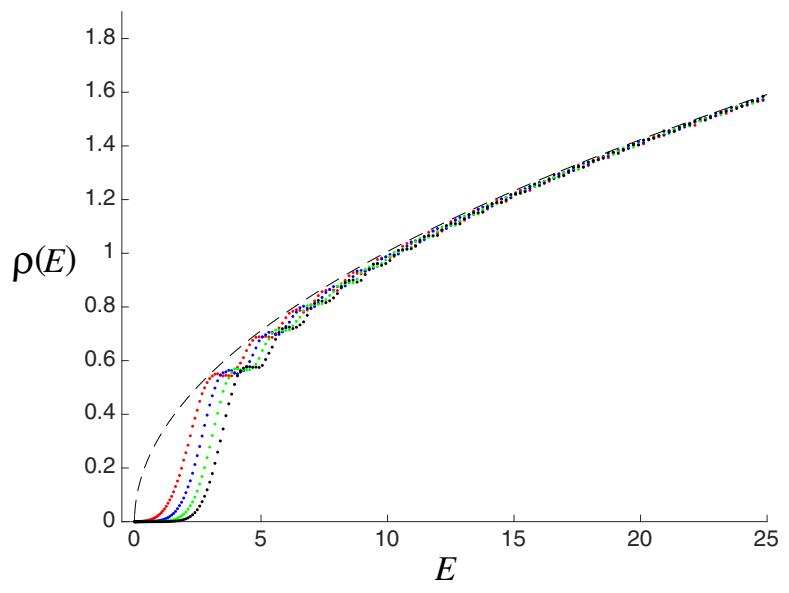

FIG. 9. The $k=1$ spectral density for $\Gamma=\frac{3}{2}, \frac{5}{2}, \frac{7}{2}$ and $\frac{9}{2}$, successively, beginning from left to right. 
$\Gamma<0$, the low- $E$ behavior is divergent. Figure 9 shows the case of $k=1$ with $\Gamma=\frac{3}{2}, \frac{5}{2}, \frac{7}{2}$, and $\frac{9}{2}$ superimposed.

It is likely that these cases (and the full JT supergravities built out of them) can be generated from the $\Gamma=\frac{1}{2}$ case by the transformation discovered in Ref. [59], that changes $\Gamma$ by an integer via a special "Bäcklund" transformation. (That work also noticed several peculiar properties of $\Gamma<0$ that might be relevant here.)

\section{DISCUSSION}

As models of 2D quantum gravity, minimal string theories (defined by the double-scaling limit of random matrix models [18-21]) produced a great deal of excitement 30 years ago because they captured, in a very compact manner, both the perturbative and nonperturbative physics of the dynamical topology of spacetime (the world sheet of the string). The beautiful demonstrations [17,33] that JT gravity and supergravity can be written as double-scaled matrix models have renewed a lot of interest in the topological dynamics of 2D gravity, but the results (expressed through recursion relations connecting topologies) are intrinsically perturbative (mostly). The work presented in this paper (and a recent earlier one [30]) is based on the idea that building JT gravity and supergravity out of minimal string models allows the powerful techniques of the older framework to be used as a complement to the recursive approach, and, moreover, helps to define the nonperturbative sector. (Note that a relation to minimal strings was suggested in Ref. [17], and another in Ref. [29]. Section II of this paper argues that the two suggestions are complementary.)

The results of this paper show that the type-0A minimal string models are the ideal components with which to build the JT supergravities that were classified in Ref. [33] as being in the $(2 \Gamma+1,2)$ Altland-Zirnbauer class. The precise recipe for combining them was found, which yields the disc-level result from the super-Schwarzian approach. Key nontrivial features of the perturbative physics were reproduced (and, in fact, made manifest in the minimal string formalism). The construction provides a stable and (probably) unique nonperturbative completion of the models, while pointing to new interesting features beyond the cases $\Gamma=0, \pm \frac{1}{2}$ (see below). (A companion paper [66] explores this construction further, solving the interpolating string equation and computing several physical quantities.)

The technique used in this paper can probably be extended in a number of directions. On the one hand, it would be interesting to formulate other quantities [correlation functions of $Z(\beta)$, the spectral form factor, etc.] in this language, thus opening a useful new window on their physical properties. On the other hand, formulating the wider set of JT gravities and supergravities (as classified in
Ref. [33]) ought to be possible. One difficulty might be that it is not clear if all the double-scaled matrix models of interest (and hence the minimal strings) have an associated Hamiltonian analogous to the one discussed in this paper [i.e., Eq. (3)], where $u(x)$ is supplied by a string equation. Its presence plays a central simplifying role in the construction. In many cases, the analogue of $u(x)$ is a combination of two or more functions, with coupled string equations linking them. The operator that, when doublescaled, becomes $\mathcal{H}$ in one-cut Hermitian, complex, and unitary matrix model cases does not seem to yield a suitable $\mathcal{H}$ in those more general cases. (See, e.g., Refs. $[67,68]$ for more on this issue of relevance to the $\beta=1,4$ Dyson-Wigner cases.) Nevertheless, perhaps even in such cases a simple effective $\mathcal{H}$ for which the spectral problem matches that of the JT system can be found. In fact, the result that the various random matrix ensembles each have an associated JT gravity encourages the conjecture that such an $\mathcal{H}$ must exist. However, there is no guarantee that it emerges at the level of individual minimal string models. That could just be a happy circumstance in the cases discussed in this paper. Finally, there were a number of results and observations in the body of the paper that hinted at larger structures that are worth further investigation. For example,

(1) In Sec. II, in determining the combination of minimal models that yields the disc spectral density, the version of the function that went into the integral transform [Eq. (9)] was a simple (and striking) generalization of that for ordinary JT gravity: $I_{1}(s) / s$ vs $I_{0}(s)$, where $s=2 \pi \sqrt{u_{0}}$, where $u_{0}$ is the classical potential and $I_{n}(s)$ is the $n$th modified Bessel function of $s$. Perhaps there is a generalization $\left[I_{n}(s) / s^{n}\right.$ suggests itself] that plays a role in defining other kinds of JT or JT-like systems.

(2) This paper's definition of JT supergravity by using component minimal models yielded (rather naturally because of the structure of the string equation) key properties of the $(0,2)$ and $(2,2)$ models that had been observed in ref. [33]. It is clear that it also supplies a definition for a wider class of models: $(2 \Gamma+1,2)$. It would be interesting to explore more properties of these, seeing if they are on at least equal physical footing to the cases of $\Gamma= \pm \frac{1}{2}$, for example.

The fact that other half-integer $\Gamma$ cases can be reached by acting with the Bäcklund transformation derived in Ref. [59] [which explicitly gives the potential $u(x ; \Gamma \pm 1)$ if $u(x ; \Gamma)$ is known] seems worth studying in this context. Perhaps the transformation has an interpretation as inserting the R-R punctures of Ref. [33]. A connection seems natural: In Ref. [59], it was pointed out that (since Bäcklund transformations change a solution's soliton number, in the associated $\mathrm{KdV}$ context) $\Gamma$ is to be associated 
with a special class of-it turned out-zero-velocity solitons present in the associated integrable system. In addition to the observation made toward the end of Sec. IV B, this connects $\Gamma$ nicely to the index $\nu$ counting additional zero-energy states in Ref. [33].

(3) Additionally, the fact that the string equation (21) has a rich family [54] of rational function solutions for half-integer $\Gamma$ may well have an application in the study of JT systems.

It is hoped that these and other issues and ideas will yield useful results to be reported soon.

\section{ACKNOWLEDGMENTS}

C. V. J. thanks Felipe Rosso for questions and conversations; Robie Hennigar, Krzysztof Pilch, Douglas Stanford and Andrew Svesko for questions and remarks; Edward Witten for drawing his attention to issues in JT gravity nine months ago; and all of the students in the USC High Energy Theory group for their willingness to learn a bit about "the old ways" of matrix models. C. V. J. also thanks the U.S. Department of Energy for support under Grant No. DE-SC 0011687, and, especially during the pandemic, Amelia for her support and patience.
[1] R. Jackiw, Nucl. Phys. B252, 343 (1985).

[2] C. Teitelboim, Phys. Lett. 126B, 41 (1983).

[3] A. Fabbri, D. Navarro, and J. Navarro-Salas, Nucl. Phys. B595, 381 (2001).

[4] P. Nayak, A. Shukla, R. M. Soni, S. P. Trivedi, and V. Vishal, J. High Energy Phys. 09 (2018) 048.

[5] D. Grumiller, W. Kummer, and D. Vassilevich, Phys. Rep. 369, 327 (2002).

[6] G. Sárosi, Proc. Sci., Modave2017 (2018) 001.

[7] A. Almheiri and J. Polchinski, J. High Energy Phys. 11 (2015) 014.

[8] K. Jensen, Phys. Rev. Lett. 117, 111601 (2016).

[9] J. Maldacena, D. Stanford, and Z. Yang, Prog. Theor. Exp. Phys. 12C104 (2016).

[10] J. S. Cotler, G. Gur-Ari, M. Hanada, J. Polchinski, P. Saad, S. H. Shenker, D. Stanford, A. Streicher, and M. Tezuka, J. High Energy Phys. 05 (2017) 118; 09 (2018) 2.

[11] J. Maldacena and D. Stanford, Phys. Rev. D 94, 106002 (2016).

[12] J. Engelsöy, T. G. Mertens, and H. Verlinde, J. High Energy Phys. 07 (2016) 139.

[13] S. Sachdev and J. Ye, Phys. Rev. Lett. 70, 3339 (1993).

[14] A. Kitaev, KITP seminars, April 7th and May 27th (2015).

[15] A. M. García-García and J. J. M. Verbaarschot, Phys. Rev. D 94, 126010 (2016).

[16] P. Saad, S. H. Shenker, and D. Stanford, arXiv:1806.06840.

[17] P. Saad, S. H. Shenker, and D. Stanford, arXiv:1903.11115.

[18] E. Brezin and V. A. Kazakov, Phys. Lett. B 236, 144 (1990).

[19] M. R. Douglas and S. H. Shenker, Nucl. Phys. B335, 635 (1990).

[20] D. J. Gross and A. A. Migdal, Phys. Rev. Lett. 64, 127 (1990).

[21] D. J. Gross and A. A. Migdal, Nucl. Phys. B340, 333 (1990).

[22] G. 't Hooft, Nucl. Phys. B72, 461 (1974).

[23] E. Brezin, C. Itzykson, G. Parisi, and J. B. Zuber, Commun. Math. Phys. 59, 35 (1978).

[24] B. Eynard, J. High Energy Phys. 11 (2004) 031.

[25] M. Mirzakhani, Inventiones Mathematicae 167, 179 (2006).

[26] B. Eynard and N. Orantin, arXiv:0705.3600.
[27] B. Eynard and N. Orantin, Commun. Num. Theor. Phys. 1, 347 (2007).

[28] N. Seiberg and D. Shih, C. R. Phys. 6, 165 (2005).

[29] K. Okuyama and K. Sakai, J. High Energy Phys. 01 (2020) 156.

[30] C. V. Johnson, Phys. Rev. D 101, 106023 (2020).

[31] P. Betzios and O. Papadoulaki, J. High Energy Phys. 09 (2020) 125.

[32] K. Okuyama and K. Sakai, J. High Energy Phys. 08 (2020) 126.

[33] D. Stanford and E. Witten, arXiv:1907.03363.

[34] A. Altland and M. R. Zirnbauer, Phys. Rev. B 55, 1142 (1997).

[35] W. Fu, D. Gaiotto, J. Maldacena, and S. Sachdev, Phys. Rev. D 95, 026009 (2017); 95, 069904(A) (2017).

[36] T. Li, J. Liu, Y. Xin, and Y. Zhou, J. High Energy Phys. 06 (2017) 111.

[37] T. Kanazawa and T. Wettig, J. High Energy Phys. 09 (2017) 050.

[38] S. Forste and I. Golla, Phys. Lett. B 771, 157 (2017).

[39] J. Murugan, D. Stanford, and E. Witten, J. High Energy Phys. 08 (2017) 146.

[40] F. Sun and J. Ye, Phys. Rev. Lett. 124, 244101 (2020).

[41] D. Stanford and E. Witten, J. High Energy Phys. 10 (2017) 008.

[42] T. R. Morris, Report No. FERMILAB-PUB-90-136-T.

[43] T. R. Morris, Nucl. Phys. B356, 703 (1991).

[44] S. Dalley, C. V. Johnson, and T. Morris, Nucl. Phys. B368, 625 (1992).

[45] S. Dalley, C. V. Johnson, and T. Morris, Nucl. Phys. B368, 655 (1992).

[46] S. Dalley, C. V. Johnson, and T. Morris, Nucl. Phys. B, Proc. Suppl. 25A, 87 (1992).

[47] I. R. Klebanov, J. Maldacena, and N. Seiberg, Commun. Math. Phys. 252, 275 (2004).

[48] T. Banks, M. R. Douglas, N. Seiberg, and S. H. Shenker, Phys. Lett. B 238, 279 (1990).

[49] P. H. Ginsparg and G. W. Moore, in Theoretical Advanced Study Institute (TASI 92): From Black Holes and Strings to Particles (1993), pp. 277-469 [arXiv:hep-th/9304011].

[50] V. A. Kazakov, Mod. Phys. Lett. A 04, 2125 (1989). 
[51] M. R. Douglas, Phys. Lett. B 238, 176 (1990).

[52] S. Dalley, C. V. Johnson, T. R. Morris, and A. Watterstam, Mod. Phys. Lett. A 07, 2753 (1992).

[53] I. M. Gel'fand and L. A. Dikii, Russ. Math. Surv. 30, 77 (1975).

[54] C. V. Johnson, arXiv:hep-th/0610223.

[55] C. V. Johnson, J. High Energy Phys. 12 (2004) 072.

[56] J. E. Carlisle, C. V. Johnson, and J. S. Pennington, J. Phys. A 41, 085401 (2008).

[57] T. Nagao and K. Slevin, J. Math. Phys. (N.Y.) 34, 2075 (1993).

[58] C. A. Tracy and H. Widom, Commun. Math. Phys. 161, 289 (1994).

[59] J. E. Carlisle, C. V. Johnson, and J. S. Pennington, J. Phys. A 40, 12451 (2007).

[60] S. P. Hastings and J. B. McLeod, Arch. Ration. Mech. Anal. 73, 31 (1980).
[61] C. V. Johnson, T. R. Morris, and A. Watterstam, Phys. Lett. B 291, 11 (1992).

[62] C. Crnkovic, M. R. Douglas, and G. W. Moore, Nucl. Phys. B360, 507 (1991).

[63] A. Watterstam, Phys. Lett. B 263, 51 (1991).

[64] M. R. Douglas, N. Seiberg, and S. H. Shenker, Phys. Lett. B 244, 381 (1990).

[65] M. Pillai, J. Goglio, and T. G. Walker, Am. J. Phys. 80, 1017 (2012).

[66] C. V. Johnson, following paper, Phys. Rev. D 103, 046013 (2021).

[67] E. Brezin and H. Neuberger, Nucl. Phys. B350, 513 (1991).

[68] E. Brezin and H. Neuberger, Phys. Rev. Lett. 65, 2098 (1990). 